ISSN 2693-2504

\title{
Surfactal Software-Cad for Comparison-Dissection of Lumbar Spine Cadaveric Specimens with Biomedical Applications
}

Journal of Bioscience \& Biomedical Engineering

Research Article

Francisco Casesnoves

PhD Engineering, MSc Physics, MD. Independent Research Scientist Engineering-Physics-Software and Medical Bioengineering Devices. IAAM (International Association of Advanced Materials). COE Tallinn University of Technology, Tallinn, Harjumaa, Estonia

\author{
*Correspondence author \\ Francisco Casesnoves \\ Independent Research Scientist Engineering-Physics- \\ Software and Medical Bioengineering Devices. \\ IAAM (International Association of Advanced Materials). \\ COE Tallinn University of Technology \\ Tallinn \\ Harjumaa \\ Estonia
}

Submitted : 24 Feb 2021 ; Published : 20 Mar 2021

\begin{abstract}
The surfactal Computer Aided Design (CAD) and Computer Aided Manufacturing (CAM) constitute a modern method with multiple biomedical applications. This article presents a comparative-dissection study with different anterior lumbar spine cadaveric specimens, based on previous contribution advances. Two new unalike specimens are selected for CAD-CAM-Specimen 1 and Specimen 2. Improved programming for lumbar spine CAD is developed from former publications. Objective results demonstrate the amount of biomedical-surgical practical/ functional that can be obtained from computational surfaces of these anatomical samples. The selected cadaveric specimens 1 and 2 are evaluated and compared anatomically and functionally after software implementation. The programming-software method(s) explanations with series of demonstrative images constitute the formal surfactal-computational resulting part. The designed computational optimization program for anatomical imaging of specimens is proven be efficacious. Consequent anatomical deductions for surgical applications are got from every image, by using appropriate subroutines and designed patterns. Out coming demonstration of the particular anatomy for any individual is determined. The singular-patient difficulties that can be found at tools manufacturing and/or surgical theatre intervention are verified. Explanations of optimization applications in future biomedical surgery, surgical robotics integrated systems, and tools manufacturing form an important part of results. Projection to surgical robotics, spinal prostheses, orthopedics, spine-rehabilitation apparatus and Forensic Robotics [Casesnoves, 2020] constitute concrete consequences.
\end{abstract}

Keywords: Software Engineering, Surfactal Programming, CAD (Computer Aided Design), CAM (Computer Aided Manufacturing), ROI, Anatomical Cadaveric Simulations, Biomechanics, Bioengineering, Spinal Computational-Surgery, Spinal Ligaments, Spinal Anterior Longitudinal Ligament (ALL), Surgery Robotics Integrated Systems.

Introduction

The spinal surgery sub-specialization in traumatology constitutes a rather complicated one for a number of reasons. Human spine was subject of evolution along the natural biological-genetic adaptation of the quadrupedal hominoids, when the erect posture was developed $[2,14]$. At that evolutionchange threshold stage, new biomechanical constraints were set for spine balance function and flexibility - the commencing of the bipedal locomotion. The protection against impact, flexionextension, bending, and rotation, became essential [14].

The peripheric nervous system is canalized and shielded by the spine. Both the cranial fundamental nerve roots, symphatetic cord ganglia, among several other crucial anatomicalphysiological structures and caudal lumbar nerve roots depend on the spinal biomechanical system around. Therefore, if any pathological trauma/disease happens in this vital system, consequences for normal life of the patient could be rather serious. Hence, the biomedical science has focused its efforts in this research field centuries ago. Surgery techniques, methods, and rehabilitation techniques have evaluated significantly in recent times.

Surfactal Computer Aided Design (CAD) and Computer Aided Manufacturing (CAM) methods have added advances in this investigation area $[7,8]$. These are both theoretical and practical. The principle of minimum invasive surgery obtains advantages/ advances from CAD and CAM. The statistical study of vertebral shape at different stages of life can provide data for surgical theatre precise instrumentation manufacturing - and also for evaluation of incidence/prevalence of spinal pathologies. The design of new prostheses and spinal screws, artificial disks, stabilization permanent/temporal, gets improvements from

J B \& Bio Engine; 2021 www.unisciencepub.com Volume 2| Issue 1 
CAD database. For instance, stabilization instrumentation, such as constrained/unconstrained stabilization connectors. These could be varied, for example, screw/grip/bolt-plate connectors or locking mechanisms in general.

Therefore, this contribution deals with new/different anterior lumbar spine cadaveric specimens for CAD-CAM, starting from previous studies $[7,8,13]$, in general references about lumbar spine all]. The primary objective is demonstrate the amount of biomedical-surgical information that can be obtained from computational surfaces of these anatomical samples. Then, the selected cadaveric specimens are compared after software-imaging is set. Anatomical deductions and specific anatomy findings, for surgical applications, are got from every image by using appropriate subroutines. A brief of future biomedical surgery, surgery robotics was presented. Darwin spinal evolution pre-hypotheses are explained/justified with biomechanical-physics base. Projection to in-future surgical robotics [16], and Forensic Robotics [Casesnoves, 2020] are included and detailed.

In summary, this contribution firstly shows computational methods for 3D image setting in biomedical CAD and CAM. Secondly, projections towards future applications, instrumentation design, and surgical robotics are overviewed. Imaging results are acceptable and evidence of bioengineering utility of results is proven.

\section{Anatomical Materials and Computational Methods}

Two lumbar spinal specimen cadaveric cloud data were selected. Cloud-3D spatial points were obtained with a 3D Scanner Digitizer (high resolution $\simeq 10 \mathrm{exp}-7 \mathrm{~m}$ ). The data obtained of the spine surfaces had $\simeq 10500$ points for each specimen. The initial point number is reduced from $\simeq 10 \exp 5$ to $\simeq 10 \mathrm{exp} 4$ to increase the visualization program speed. For the cadaveric specimen of this study, number of surfactal points are approximately 1700 (Specimen 1) and 1450 (Specimen 2).
The study is focused on the new programming method design, $[7,8]$, the new anatomical information that can be obtained from the anterior lumbar spine, and modern biomedical and robotics applications. Therefore, the programming method was based on both subroutines and a group of codes to set spinal images accurately. One another are complementary and functionally improved. It was rather complicated the setting of around 2000 cloud points and at the same time to obtain a running time short. These images are screened in at least 5-7 seconds. However, this difficulty was sorted mainly with two methods. Programming techniques are based on previous publications, $[7,8,13]$. First, selecting the fastest subroutines for $3 \mathrm{D}$ imaging, among several available. Second, setting optimal pattern/order for the program codes to sort/save unnecessary mathematical operations, loops, and reiterations of calculus.

The average running time for a cadaveric image was 5-7 seconds. Specimen 2 takes longer time as it has about 300 cloud points more. The image tools time to start to work in different views/angles was about 4 seconds. The division of numerical intervals for Voronoi and Delaunay tiles was selected about 0.5 for optimal visualization and not too long running time. In summary, this original software-engineering method, [Casesnoves, 2020-2021] was rather difficult for obtaining precision.

\section{Results Cadaveric Specimen 1}

The implementation of CAD software results is explained in a series of images. At every image, the biomedical- anatomical study is detailed. Specimen 1 is an anatomical sample whose image can be considered acceptable for obtaining data. In all specimens, there are rounded deformations at disks that were done for other research, and do not have any significance here. However, they are useful to perfectly identify the location of every disk.

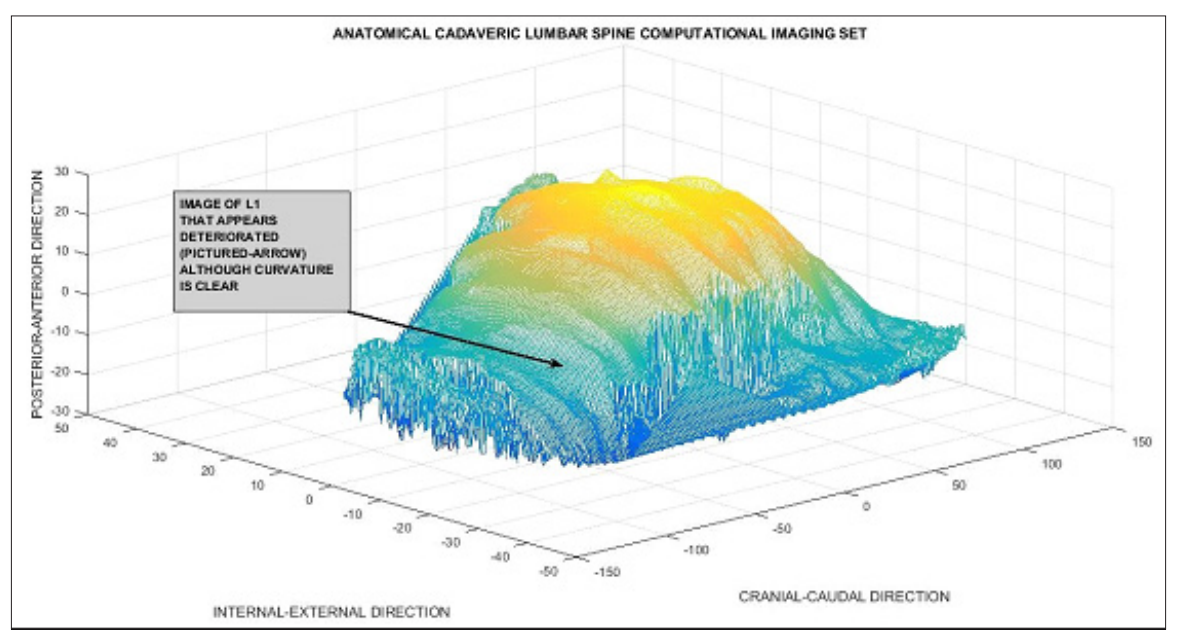

Figure 1: Cranial view of specimen 1. The L1 vertebra appears being deteriorated. However, curvature of anterior face identifies 


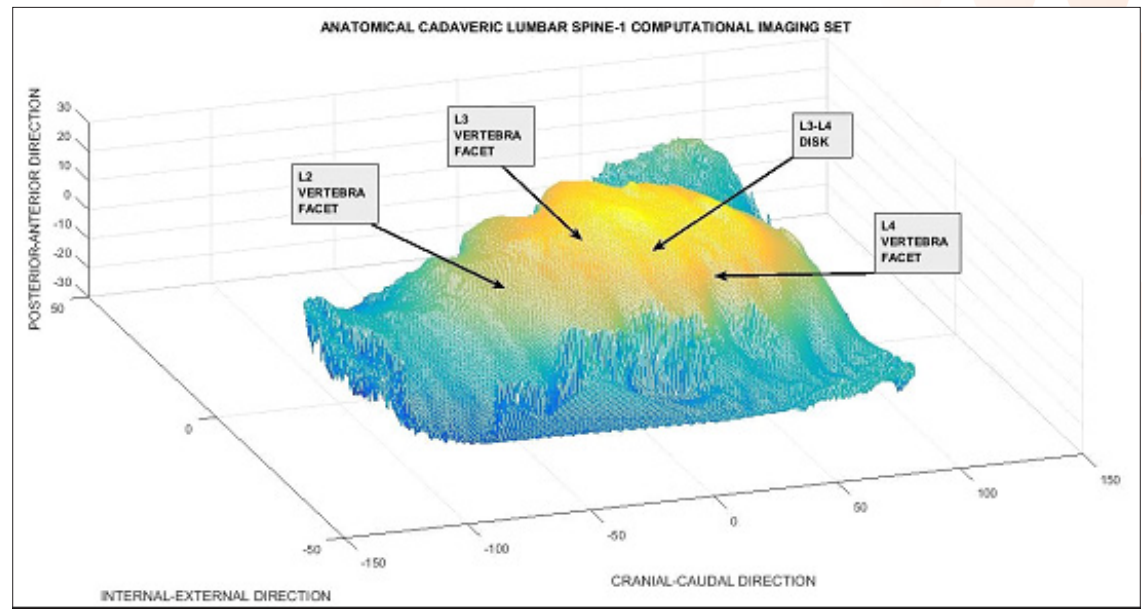

Figure 2: -Complete anatomical determination of Specimen 1. Lateral-right view. Between L2 and L3 the traces of ALL can be identified [13].

Results Cadaveric Specimen 2

Second specimen can be considered worse for study compared to Specimen 1. However, this constitutes an advantage for demonstration of diversity of patient anatomy subject to surgery, especially when age and physical activity have modified the bone surface and structure. Applications of these data therefore are useful for prostheses and orthopedic design, because the lifetime expectations have increased significantly in this century. Figures 3,4 show imaging results for Specimen 2.

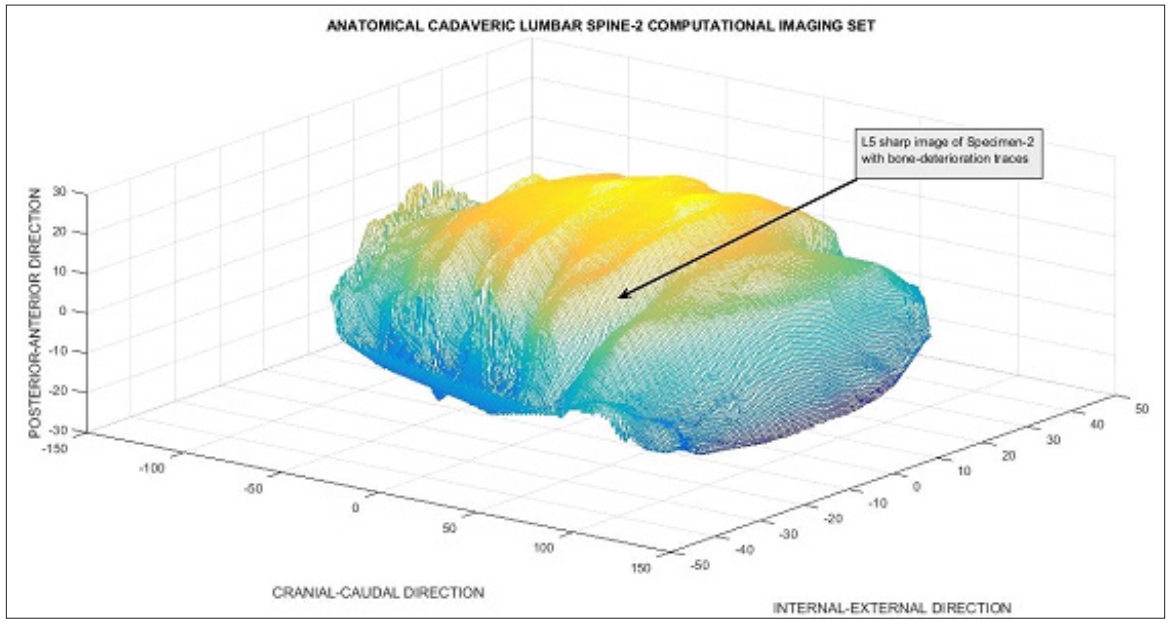

Figure 3: - Specimen 2 caudal view showing profile of L5. The vertebral body bone is worn due to age. On the right, the L5-S1 disk. This cadaveric specimen is worse in general compared to Specimen1.

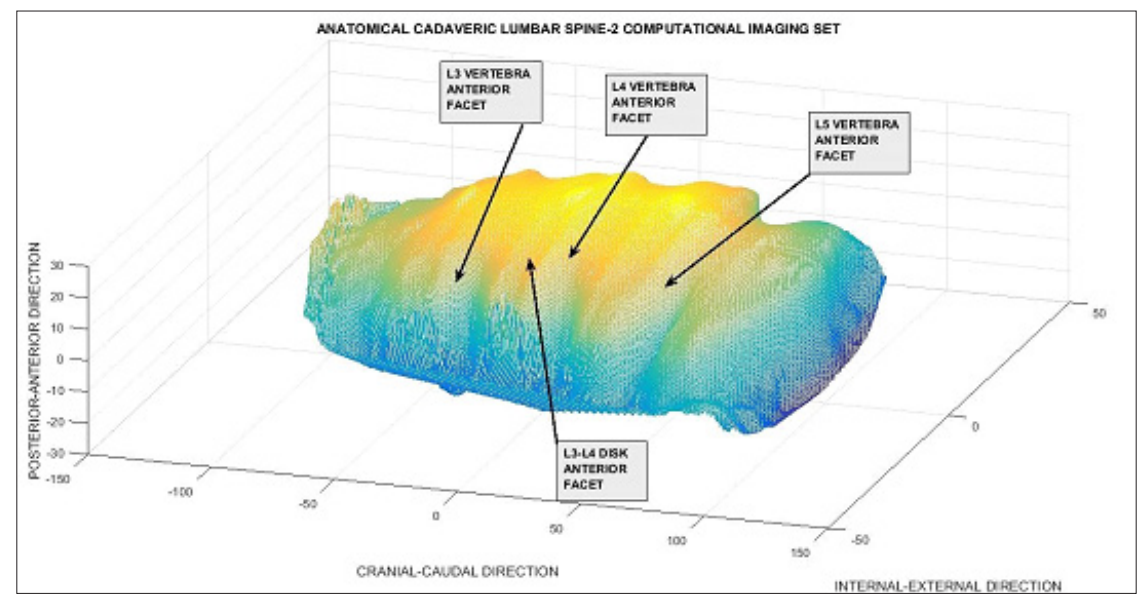

Figure 4:-Complete anatomical determination of Specimen 2. Lateral-right view. L5 size makes it easily visualizable. 
Computational Dissection Software for Cadaveric Specimens

This section deals with programming methods and subsequent imaging results of computational dissections. The objective is to rule out how to make CAD anatomical dissections with the implemented spine images. In other words, to separate selected vertebras, disks, or parts/sections of the cadaveric lumbar spines by using special software. In such way it is possible to study in detail the selected parts with computational dissection.

The new programming method designed was done by setting segmentation-planes for the dissection of selected parts. The code for setting the dissection planes involves rather difficult patterns. In Figure 5 the Specimen 1 L3 dissection is shown. Figure 6 the Specimen 2 dissection result for L5 is detailed.

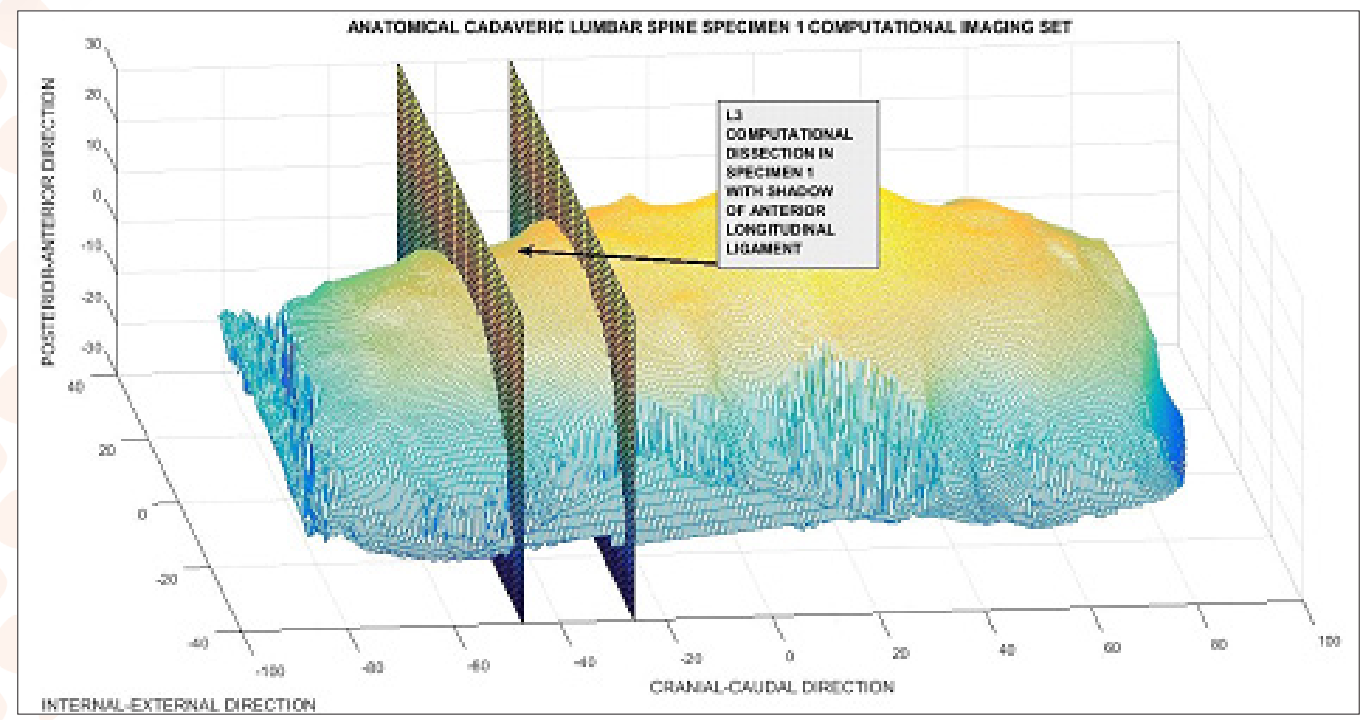

Figure 5: -Computational dissection of L3 vertebral body in Specimen 1. Dissection planes are set to define the structure of L3. The curvature and concavity of L3 is clearly defined. It can be observed the ALL ligament shadow. At level of L5 can be identified the emerging section of the vertebral body pedicle. The pedicles of L5 differ in shape compared to other lumbar vertebra pedicles. Usually, L4 is the biggest among the lumbar vertebras.

The segmentation of the selected parts also can be chosen at any anatomical direction by dissection planes. Segmentation is useful for a number of purposes - just to cite some examples. It is possible to measure correctly the size of every vertebra, disks, or vertebra group with corresponding disks. For comparison of specimen sizes, to guess precisely the degradation of the dissected zone. In the primary stages for computational CAD manufacturing of tools, to overview the geometry that could fit better at the tool parts where the force(s) are applied.

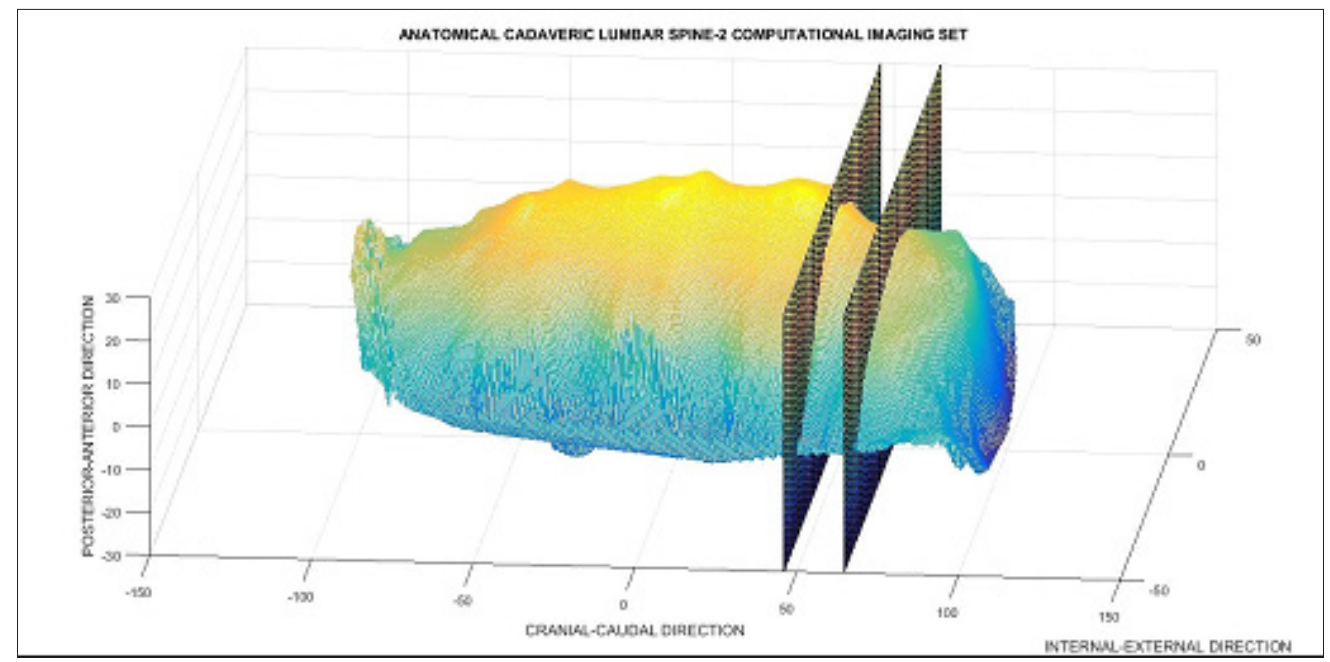

Figure 6: - Computational dissection of L5 vertebral body in Specimen 2. Dissection planes are set to define the structure of L5. The curvature and concavity of L5 is clearly defined. 
Comparative Anatomical-Biomechanical Study

In Table 1, the similarities/differences between cadaveric specimens are detailed. The study was performed in several imaging positions. The patient of Specimen 2 is probably from a taller and bigger corpse than patient of Specimen 1.

\begin{tabular}{|l|l|l|l|l|}
\hline COMPARATIVE ANATOMICAL-BIOMECHANICAL STUDY \\
\hline CHARACTERISTIC & SPECIMEN 1 & SPECIMEN 2 & INTERRELATION & $\begin{array}{l}\text { BIOMEDICAL } \\
\text { CONSEQUENCES }\end{array}$ \\
\hline GENERAL IMAGE & $\begin{array}{l}\text { good more } \\
\text { curvature than } 2\end{array}$ & less good & specimen 2 longer & anatomical diversity proof \\
\hline VERTEBRA SHAPE & good & less good & specimen 2 wider & anatomical age degeneration proof \\
\hline DISK SHAPE & good & good & $\begin{array}{l}\text { specimen 2 longer/ } \\
\text { wider }\end{array}$ & $\begin{array}{l}\text { Patient spine 2 probably older with } \\
\text { higher height and weight }\end{array}$ \\
\hline $\begin{array}{l}\text { VERTEBRAL } \\
\text { BONE-SURFACE }\end{array}$ & deformed by age & $\begin{array}{l}\text { deformed by age, } \\
\text { less concave }\end{array}$ & $\begin{array}{l}\text { specimen } 1 \text { better } \\
\text { conservation }\end{array}$ & $\begin{array}{l}\text { vertebras more degenerated due to } \\
\text { patient higher weight and height }\end{array}$ \\
\hline $\begin{array}{l}\text { AGEING } \\
\text { DETERIORATION }\end{array}$ & normal-high & higher & $\begin{array}{l}\text { probable similar } \\
\text { patient age }\end{array}$ & $\begin{array}{l}\text { not determinated probably } \\
\text { specimen 2 older }\end{array}$ \\
\hline $\begin{array}{l}\text { PEDICLE } \\
\text { VISUALIZATION }\end{array}$ & $\begin{array}{l}\text { observable L5, } \\
\text { L4 }\end{array}$ & observable L5 & $\begin{array}{l}\text { smaller spine 1 } \\
\text { difficult to guess }\end{array}$ \\
\hline $\begin{array}{l}\text { LIGAMENT } \\
\text { VISUALIZATION }\end{array}$ & observable & observable & $\begin{array}{l}\text { sharp in both } \\
\text { specimens }\end{array}$ & ligaments well state despite age \\
\hline
\end{tabular}

Table 1: Anatomical comparative study of specimen images. Differences and similarities are highlighted. It is important to clarify that all these differences/similarities were found/guessed with imaging tool visualization at several positions.

\section{Bioengineering and Biomechanical Applications}

The applications of the computational methods and imaging results presented are varied. CAD and CAM in traumatology and orthopedics in general can be used for tool manufacturing, apparatus, prostheses, artificial implants fitting, and industrialmedical devices $[1,7,8,13]$. In particular, for spinal surgery, artificial disk technology, spinal stabilization technology, rehabilitation apparatus, and a wide range of related options. Precision implies efficiency at surgical theatre, savings of time, improvements to avoid post-operational complications, and patient quality of life. That is, restoration to normality and bipedal locomotion.

From the biomechanical point of view [1], the efficiency and efficacy of instrumentation/prostheses/implants are function of several variables. Utility for the forces that will be exerted, precision to fit the surfaces/parts where the force is applied, capability to have dynamic options during the task, multifunctionality for reduction of instrumentation excess, and histocompatibility for permanent/temporary implants/ prostheses. In particular, the widely-used screws in spinal surgery requires a number of biomechanical conditions. Insertion angle is very important, and can be statistically calculated with CAD and/or CAM. If screws are permanent, histocompatibility is a must-bioabsorbable screws or bioabsorbable spinal cages, for example, is an alternative. Screws are considered effective in cervical spine surgery, but not in general for the lumbar spine (e. g., Spondylolisthesis surgery). This is caused by the different magnitudes of the biomechanical forces corresponding to each spine level.

Pediatric surgery is even more complicated in general than the adult's one. The children organism and constitution is in dynamic-growing continuous evolution. This difficulty implies that prostheses or artificial implants could have a short time of utility, unless that adaptable implants/prostheses are set. The tissular viscoelasticity and bone hardness also changes in time during the children's growth stages. Apart from that the physical/sporting/gaming activity of kids is higher compared to adults. Computational Biomechanics design constitutes a useful tool to rule out these pediatric biomedical problems.

Prospective Surgical Robotics Applications and Brief of Human Spine Evolution

Approximately $20 \%$ of surgical robots are designed for orthopedics surgery [16]. Robotic integrated systems perform better when structures for force exertions are solid (like bone) or semi-rigid (viscoelastic lumbar tissues). Furthermore, infection probability is reduced, CT,MRI,RX,SPECT contrast is better, and radioprotection dose-limits (exposition, scatter radiation, and absorbed dose) for staff is reduced. Artificial intelligence is currently being implemented in robot integrated systems.

Additionally, Forensic Robotics [Casesnoves, 2019] concepts may be useful in future. Advantages would be that monitoring or anesthesia are unnecessary, the absence of risks for patient life if failure happens, and the time schedule without constraints. CAD and CAM spinal simulations are therefore useful for minimal invasive robotic surgery, setting of special prostheses that require extreme precision, pre-operative spinal planning, and simulation/training of pre-operative spinal surgery. Pedicular screws are difficult to be inserted, because the pedicles are curved, have rounded geometry, and the hardness is lower than the vertebral body. Therefore, robotic systems could get a reduction of failure rate [16]. Veterinary robotics surgery concepts [Casesnoves, 2020], could constitute 
a source of future-advance in the surgical robotics area also.

In the following, a number of biomechanical pre-hypotheses and ideas are presented/explained. The objective is to demonstrate the importance of spinal evolution for getting practical-tangible advances in surgery and spinal surgery. The evolution of human spine from primates is a rather difficult part of the human Darwinist evolution $[2,14]$. This is justified for an important number of reasons. The main is the erectbipedal posture that arose the difference between primates and hominoids. When getting on the standing up vertical posture, the biomechanical changes resulted significant. The gravity center of body is elevated and centered down to the abdomen (usually at S2 level). The spine can vertically be bent, rotated or rotated-bent, which is a biomechanical difference related to quadrupeds/tetrapods. In tetrapods the load on spine points towards the ground, and its load direction is lateral related to the spine-line. The hominoids adaptation-evolution to standing up posture changed abruptly this load direction for a cranialcaudal vertical load pointing towards hip-femur frame [2,14].

Therefore, the hominoids evolution resulted in a decrease of the number of vertebras and length of spine. The evolutionary reason is mainly biomechanical. In other words, a shortened spine with less number of vertebras shows less possibilities to be bent, make the walk more difficult, or being injuredmainly during run, walk, or physical activity. Provided the spine is shorter, the momentum magnitude [F x d] at any point is lower. For instance, spinal scoliosis and similar pathologies show higher incidence and prevalence in tall individuals with longer spines. That is, for lumbar spine the biomechanical moment has a longer arm from the gravity center to the lumbar spine in individuals with longer spine. Then the magnitude of the thorax-abdomen bending moment over the spine increases and can damage the lumbar disks and vertebras easier.

The genetic evolution marked the difference in height among individuals mainly by femur length. The hypothetical reasons are to avoid longer spines, keep the gravity center in lowabdomen (about S2), and get longer legs for better- faster walk and run. In addition, the erect posture provided the hominoids with wider panoramic visual field in hunting tasks, run, walk, or defense for survival [14]. The bipedal locomotion showed for survival of the specie more advantages than inconvenient.

Furthermore, the loads in spine, and especially on lumbar vertebras, increased up to about $500 \mathrm{~N}$ when quadrupedal hominoids developed the standing up posture. This caused the rate-growth in prevalence of lumbar disk and lumbar spine pathologies/deformations in humans. Therefore, the consequences for hominoids when the standing up and vertical walk was evolutionarily developed are many and diverse.

In summary, all these evolutionary different characteristics in humans related to primates justify the study of lumbar spine to find the solution for newly created pathological probabilities/ possibilities. CAD and CAM to resolve, simulate and optimize the spinal surgery techniques is important. Computational design of surgical instrumentation, orthopedics apparatus, artificial disks, spinal screws, clamps, or similar tools get benefit for useful/practical solutions.

\section{Discussion and Conclusions}

The results of this study show the comparative-imagingprogramming method to obtain accurate surfactal images from scanning cloud data of two selected/different cadaveric specimens of lumbar spine. Image sharpness and details can be considered acceptable at anterior lumbar spine facets.

The real anatomical model of the vertebral, anterior longitudinal ligament, some pedicles and disks can be visualized. Intervertebral disks borders and surface-separation among them and vertebras are clear. In general, ageing of cadaveric specimens constitutes a proof of the rather complicated biomechanical system in human standing-up evolution.

The software used with subroutines complemented with a specific program has been developed-improved from previous contributions. Lateral parts of the spine had not sufficient cloud data to be drafted correctly, because of the source-angle of scanning waves. The second type of programs were designed to dissect with segmentation-planes selected vertebras and disks. The aim of this special software is to visualize detailed profile for any, ad libitum, selected vertebra(s) and/or disks(s), or segment/part. The anatomical differences/similarities between the two specimens are contrasted/differentiated.

This method applications of geometrical database, such as curvatures, concavities depth, angles of curvature, size of vertebral bodies, and degeneration of vertebras with elderly specimens are useful for surgical industry. These purposes are suitable for precise surgical spinal tools and stabilization devices manufacturing. A wide range of instrumentation gets advantage of these programming techniques. Clamps, screws, rip, or bolt-plate connectors can be improved-either constrained or unconstrained. Surgical robotics for future and computational preparation/design of intervention may well get advances. Post-operation patient control can also be developed with this statistical database that could predicts complication probabilities at any post-intervention stage. Therefore, with sufficient time for an anticipated surgical/clinical solution. A few of Forensic Robotics engineering concepts, [Casesnoves, 2020], were presented in Surgical Cybernetics section. Biomechanical pre-hypotheses to support the utility/efficacy of the study based on hominoids evolution were succinctly explained [2,14]. The projection of these pre-hypotheses about spinal biomechanical Darwinist-evolution in humans provide ideas for future bioengineering advances of spinal surgery.

In brief, this paper has demonstrated how imaging information can be obtained from specimens in order to be used with biomedical applications and in surgical-medical industry. Future spinal surgery applications and spine evolution prehypotheses concepts are included. 
Scientific Ethics Standards

This contribution is based on Graphical VisualizationOptimization methods for cadaveric specimens of lumbar spine with software improved from previous articles. GraphicalOptimization Methods were created by Francisco Casesnoves on December 2016. Forensic Robotics Integrated Systems engineering-concepts were developed by Casesnoves in July 2020. The software to plane-separation of vertebras and disks was originally developed by author. This advanced article has a few previous paper information, whose inclusion is essential to make the contribution understandable. This study was carried out, and their contents are done according to the European Union Technology and Science Ethics. Reference, 'European Textbook on Ethics in Research'. European Commission, Directorate- General for Research. Unit L3. Governance and Ethics. European Research Area. Science and Society. EUR 24452 EN [17,18]. This research was completely done by the author, the software, calculations, images, mathematical propositions and statements, reference citations, and text is original for the author. When anything is taken from a source, it is adequately recognized. Ideas from previous publications were emphasized due to a clarification aim, [17,18].

\section{References}

1. Panjabi, M, White, A (1980) Clinical Biomechanics of the Spine. Lippincott. 1980.

2. Ben, E, Gomez-Olivencia, A, Kramer,P (2019) Spinal Evolution. Springer. 2019.

3. Casesnoves, F (2019) The Numerical Reuleaux Method, a computational and dynamical base with applications. First Part. Lambert Academic Publishing. ISBN-10 3659917478. 2019.

4. Casesnoves, F (2019) Die Numerische ReuleauxMethode Rechnerische und Dynamische Grundlagen mit Anwendungen (Erster Teil). ISBN-13 : 978-620-0-895608, ISBN-10: 6200895600. Publishing House: Sciencia Scripts. 2019-20.

5. Casesnoves F, Suzenkov A (2017) Mathematical Models in Biotribology with 2D-3D Erosion Integral-Differential Model and Computational-Optimization/Simulation Programming. International Journal of Scientific Research in Computer Science, Engineering and Information Technology. 2(3) : 2456- 3307.

6. Casesnoves F (2018) Mathematical Models and Optimization of Erosion and Corrosion. Taltech University Press. Doctoral Thesis. ISSN 25856898. 2018.

7. Casesnoves, F (2012) 'Computational Simulations of Vertebral Body for Optimal Instrumentation Design'. ASME Journal of Medical Devices (Research Paper). Author: F Casesnoves .Journal of Medical Devices. June 2012. Volume 6. Issue 2/021014.11 pages.http://dx.doi. org/10.1115/1.4006670.

8. Casesnoves, F (2015) Applied inverse methods for optimal geometrical-mechanical deformation of lumbar artificial disks/implants with Numerical Reuleaux method. 2D comparative simulations and formulation. Ethan Publishing Computer Science Applications. 2(4): 1-10.
[USA Congress Library registration].

9. Casesnoves, F (2007) 'A Monte-Carlo Optimization method for the movement analysis of pseudo-rigid bodies'. 10th SIAM Conference in Geometric Design and Computing, Texas, San Antonio, USA. Contributed Talk. November 2007.

10. Casesnoves, F (2013) 'Applied Inverse Methods for Deformable Solid Dynamics/Kinematics in Numerical Reuleaux Method (NRM)'. International Journal of Numerical Methods and Applications. 9(2): 109131.peer-reviewed International Mathematical/ Computation Journal Article. print/Online.http://www. pphmj.com/abstract/7688.htm. This article is specially innovative in Inverse Problems applications for deformable solids kinematics/dynamics, further publications are included in United States Congress Library and Numerical Reuleaux Method is accepted by scientific community as an innovative dynamics method in deformable solids with mechanical, biomechanical and aerospace applications. New applications of this method will be probably found significantly in future.

11. Casesnoves, F (2018) Nonlinear comparative optimization for biomaterials wear in artificial implants technology. Presented in Applied Chemistry and Materials Science RTU2018 Conference Proceedings. 2018.

12. Casesnoves, F (2015) Applied Inverse Methods for Optimal Deformation of Lumbar Artificial Disk/Implants with Numerical Reuleaux Method and 3D VoxelizationComputational Simulations. American Institute of Science. Bioscience and Bioengineering. 1(4): 94-105. http://www.aiscience.org/journal/bio.

13. Casesnoves, F (2012) 3D Improved mathematical model for lumbar intervertebral ligaments (LILs). Proceedings SIAM Life Sciences Conference joint to SIAM Annual Conference San Diego.pp. 25-27.

14. Darwin,C (2004) The origin of species. Barnes \& Noble Classics. 2004.

15. Todinov, M (2005) Reliability and Risk Models. Wiley. 2005.

16. Rosen, J, Hannaford, B. Satava, R (2011) Surgical Robotics. Wiley. 2011.

17. 'European Textbook on Ethics in Research'. European Commission, Directorate-General for Research. Unit L3. Governance and Ethics. European Research Area. Science and Society. EUR 24452 EN.

18. The European Code of Conduct for Research Integrity. Revised Edition. ALLEA. 2017.

Copyright: C2021 Francisco Casesnoves. This is an open-access article distributed under the terms of the Creative Commons Attribution License, which permits unrestricted use, distribution, and reproduction in anymedium, provided the original author and source are credited. 